\title{
Association between blood pressure and the risk of chronic kidney disease in treatment-naïve hypertensive patients
}

\author{
Haekyung Lee ${ }^{1}$, Soon Hyo Kwon ${ }^{1,2}$, Jin Seok Jeon ${ }^{1,2}$, Hyunjin Noh ${ }^{1,2}$, Dong Cheol Han ${ }^{1,2}$, Hyoungnae Kim ${ }^{1,2}$ \\ ${ }^{1}$ Division of Nephrology, Department of Internal Medicine, Soonchunhyang University Seoul Hospital, Seoul, Republic of Korea \\ ${ }^{2}$ Hyonam Kidney Laboratory, Soonchunhyang University Seoul Hospital, Seoul, Republic of Korea
}

\begin{abstract}
Background: Although hypertension is a well-known risk factor for chronic kidney disease (CKD), the blood pressure (BP) at which antihypertensive interventions should be initiated remains to be determined. Therefore, we investigated the association between BP and CKD in treatment-naïve individuals.

Methods: This prospective cohort study considered 7,343 individuals in the Korean Genome and Epidemiology Study who were not taking antihypertensive medications. Subjects were categorized into six groups according to their systolic BP (SBP) and five groups according to their diastolic BP (DBP). The primary outcome was incident CKD, which was defined as an estimated glomerular filtration rate of $<60 \mathrm{~mL} / \mathrm{min} / 1.73 \mathrm{~m}^{2}$ or the development of proteinuria. The secondary outcome was incident cardiovascular disease (CVD). Results: In the time-varying Cox models, the hazard ratios (95\% confidence interval [CI]) for CKD were 1.39 (1.10-1.77) with SBP 130-139 mmHg, 1.79 (1.40-2.28) with SBP 140-159 mmHg, and 3.22 (2.35-4.40) with SBP $\geq 160 \mathrm{mmHg}$, compared with SBP 100-119 mmHg. In addition, the hazard ratios (95\% Cl) for CKD were 1.88 (1.48-2.37) with DBP 90-99 mmHg and 4.30 (3.205.76) with DBP $\geq 100 \mathrm{mmHg}$, compared with DBP $70-79 \mathrm{mmHg}$. A significantly increased CVD risk was also observed in subjects with $\mathrm{SBP} \geq 130 \mathrm{mmHg}$ or $\mathrm{DBP} \geq 90 \mathrm{mmHg}$.

Conclusion: Our findings indicate that $\mathrm{SBP} \geq 130 \mathrm{mmHg}$ and $\mathrm{DBP} \geq 90 \mathrm{mmHg}$ are associated with an increased risk of CKD. Therefore, BP-lowering strategies should be considered starting at those thresholds to prevent CKD development.
\end{abstract}

Keywords: Blood pressure, Chronic kidney disease, Cardiovascular diseases, Hypertension, Incidence

\section{Introduction}

Hypertension (HTN) is a well-known risk factor for cardiovascular disease (CVD) and chronic kidney disease (CKD) $[1,2]$. The kidney is one of the major organs damaged by high blood pressure (BP), and hypertensive nephrosclerosis is the predominant cause of CKD [3,4]. Furthermore, the incidence of HTN is directly proportional to a decline in renal function $[5,6]$; thus, early detection and proper management of HTN is needed to reduce the risk of CKD and

Received: April 29, 2021; Revised: July 2, 2021; Accepted: July 7, 2021

Correspondence: Hyoungnae Kim

Division of Nephrology, Department of Internal Medicine, Soonchunhyang University Seoul Hospital, 59 Daesagwan-ro, Yongsan-gu, Seoul 04401, Republic of Korea. E-mail: hkim@schmc.ac.kr

ORCID: https://orcid.org/0000-0002-5359-0214

Copyright (C) 2022 by The Korean Society of Nephrology

(a) This is an Open Access article distributed under the terms of the Creative Commons Attribution Non-Commercial and No Derivatives License (http:// creativecommons.org/licenses/by-nc-nd/4.0/) which permits unrestricted non-commercial use, distribution of the material without any modifications, and reproduction in any medium, provided the original works properly cited. 
its related complications. In 2017, the American College of Cardiology/American Heart Association suggested a lower threshold for HTN defined as systolic BP (SBP) $\geq 130$ $\mathrm{mmHg}$ or diastolic BP (DBP) $\geq 80 \mathrm{mmHg}$ [7]. The decrease in threshold was largely influenced by the Systolic Blood Pressure Intervention Trial (SPRINT), which found that maintaining SBP $<120 \mathrm{mmHg}$ clearly reduced CVD risk [8].

Although intensive BP control can lower CVD risk and mortality, the results of large randomized controlled trials (RCTs) have raised concerns that strict BP control can produce an increased risk of CKD [8-10]. Moreover, RCTs have usually been conducted in individuals with high CVD risk who are already taking antihypertensive medications. Therefore, deciding when to begin BP control medication in individuals with a low CVD risk remains to be determined. Early BP control interventions might not reduce or could even harm renal function, but late intervention can increase the risk of CKD, CVD, and the mortality associated with those conditions. Previous observational studies have suggested that prehypertension is associated with an increased CKD risk in the general population, but those studies defined prehypertension according to a variety of criteria or included patients on antihypertensive medications [11-14].

Thus, substantial uncertainty remains regarding the best BP level for CKD prevention, particularly among individuals without underlying kidney disease who are not taking antihypertensive medication. Therefore, we examined the association between BP and incident CKD risk in a large Korean population who had not taken any antihypertensive medication.

\section{Methods}

\section{Study population}

In this study, we used data from the Korean Genome and Epidemiology Study (KoGES), a prospective community-based cohort study of people living in urban and rural areas. The study profile and methods used have already been described $[15,16]$. In brief, the KoGES study population consisted of 10,030 individuals aged 40 to 69 years. From 2001 to 2014, participants underwent serial medical examinations and completed a biennial self-administered questionnaire about their health, lifestyle, and diet. We excluded individuals with no follow-up visits $(\mathrm{n}=974)$; without baseline serum creatinine level or proteinuria data ( $\mathrm{n}=29$ ); with an estimated glomerular filtration rate (eGFR) $<60 \mathrm{~mL} / \mathrm{min} / 1.73 \mathrm{~m}^{2}$, proteinuria $1+$, or a history of underlying kidney disease at baseline $(n=625)$; taking antihypertensive medications at baseline $(n=898)$; or with a history of CVD at baseline $(\mathrm{n}=161)$. Thus, 7,343 subjects who provided written informed consent voluntarily participated in this study (Supplementary Fig. 1, available online).

This study was carried out in accordance with the ethical principles of the Declaration of Helsinki and was approved by the Ethics Committee of the Korean Health and Genomic Study at the Korea National Institute of Health and the Institutional Review Board of Soonchunhyang University Hospital (No. 2019-03-022).

\section{Data collection}

Demographic and socioeconomic data were collected at baseline. Height and body weight were measured following standard methods with the patient wearing light clothes, and body mass index (BMI) was determined as $\mathrm{kg} / \mathrm{m}^{2}$. Educational status was divided into three categories: low, lower than middle school; intermediate, middle school; and high, higher than middle school. Income status was classified into three categories: low, $<\$ 850$ per month; intermediate, $>\$ 850$ to $<\$ 1,700$ per month; and high, $>\$ 1,700$ per month. Smoking status was divided into three categories: current smokers, former smokers, and never smokers. Diabetes mellitus (DM) was defined as fasting glucose $\geq 126 \mathrm{mg} / \mathrm{dL}$, post-load glucose $\geq 200 \mathrm{mg} / \mathrm{dL}$ after a 75 -g oral glucose tolerance test, hemoglobin Alc $\geq 6.5 \%$, a medical history of DM, or the use of oral medication or insulin. Subjects with a history of dyslipidemia or using lipid-lowering medications were defined as having dyslipidemia. Blood samples were collected after overnight fasting. Urine samples were collected in the morning after the first voiding and were assessed for the presence of protein using a dipstick. Proteinuria was defined as $1+$ or greater.

\section{Subgroup category according to the systolic BP and dia- stolic BP}

Using a mercury sphygmomanometer, trained nurses measured BP after the subjects had been in a relaxed state for at least 10 minutes in a sitting position. The arm with the 
higher reading was used. The participants were divided into six categories according to SBP $(<100,100-119,120-$ $129,130-139,140-159$, and $\geq 160 \mathrm{mmHg}$ ) and five categories according to DBP $(<70,70-79,80-89,90-99$, and $\geq 100$ mmHg).

\section{Outcome measures}

The primary outcome was incident $\mathrm{CKD}$, which was defined as eGFR of $<60 \mathrm{~mL} / \mathrm{min} / 1.73 \mathrm{~m}^{2}$ or the development of proteinuria at least twice during follow-up. The eGFR was calculated using the Chronic Kidney Disease Epidemiology Collaboration equation [17]. The secondary outcome was incident CVD, which was defined as newly diagnosed or prescribed medications for myocardial infarction, coronary artery disease, congestive heart failure, cerebrovascular disease, or peripheral artery disease during follow-up.

\section{Statistical analysis}

Continuous variables are expressed as means \pm standard deviations, and categorical variables are expressed as numbers and percentages. Continuous data were tested for homogeneity of variances using Levene's test. When variances were not homogeneous, Welch's analysis of variance was used to assess differences among BP groups, with a Tukey or Games-Howell post-hoc analysis, as appropriate. The Cochran-Armitage trend test was used for categorical variables. A Kaplan-Meier analysis was used to estimate CKDfree survival, and a log-rank test was used to determine significant differences between survival curves. We used Cox proportional hazards regression analyses to determine the relationship between $\mathrm{BP}$ and incident CKD. We also investigated the influence of time-dependent covariates. Details of the variables included and the consecutive models are provided in Supplementary Table 1 (available online). The nonlinear relationship between the CKD hazard ratio (HR) and the baseline or time-varying BP was evaluated using restricted cubic spline curves. In all survival analyses, right-censoring occurred due to administrative censoring, death, loss to follow up, or the initiation of antihypertensive medication during follow-up. For all analyses, $p<0.05$ was considered statistically significant. Statistical analyses were performed using R version 4.0.2 (R Foundation for Statistical Computing, Vienna, Austria).

\section{Results}

\section{Baseline characteristics}

The baseline characteristics of the eligible participants according to their SBP categories are presented in Table 1. The mean age of the subjects was $51 \pm 9$ years, and 3,736 of them (50.9\%) were female. The mean eGFR was $94 \pm$ $13 \mathrm{~mL} / \mathrm{min} / 1.73 \mathrm{~m}^{2}$. The prevalence of DM was $5 \%$. BP was measured five times on average during the follow-up period, and the mean SBP and DBP were $122 \pm 17 \mathrm{mmHg}$ and $82 \pm 11 \mathrm{mmHg}$, respectively. Participants in the higher baseline SBP groups were more likely than others to be older and have higher BMI, lower educational levels, and lower-income levels and less likely than others to be current smokers. Moreover, the high SBP group had higher levels of serum fasting glucose, total cholesterol, triglycerides, hemoglobin, and serum albumin and lower eGFR. These trends were similar among the DBP groups (Supplementary Table 2, available online).

\section{Incidence of chronic kidney disease according to blood pressure}

During a median follow-up period of 10 years (interquartile range, 6-12 years), 603 incident CKD events (8.2\%) occurred, and the crude incidence rate was 9.67 (95\% confidence interval [CI], 8.92-10.48) per 1,000 person-years. During follow-up, 2,028 participants were censored when they started taking antihypertensive medication, and their median follow-up period was 6 years (interquartile range, 4-9 years). The CKD incidence tended to increase significantly in the higher baseline SBP and DBP groups ( $p$ for trend $<0.001$, both) (Supplementary Table 3, available online).

\section{Relationship between blood pressure and incident chron- ic kidney disease}

The Kaplan-Meier survival curves show a statistically significant difference in incident CKD between the BP groups. When comparing the groups using SBP 100-119 mmHg as the reference, the cumulative CKD-free survival rate was significantly lower in all the groups with $\mathrm{SBP} \geq 120 \mathrm{mmHg}$ (log-rank test $\mathrm{p}<0.001$ for all) (Fig. 1A). When comparing 
Table 1. Baseline characteristics of the participants

\begin{tabular}{|c|c|c|c|c|c|c|}
\hline \multirow[b]{2}{*}{ Variable } & \multicolumn{6}{|c|}{$\mathrm{SBP}(\mathrm{mmHg})$} \\
\hline & $\begin{array}{c}<100 \\
(n=397)\end{array}$ & $\begin{array}{c}100-119 \\
(n=3,016)\end{array}$ & $\begin{array}{c}120-129 \\
(n=1,628)\end{array}$ & $\begin{array}{c}130-139 \\
(n=1,148)\end{array}$ & $\begin{array}{l}140-159 \\
(n=889)\end{array}$ & $\begin{array}{c}\geq 160 \\
(n=265)\end{array}$ \\
\hline Age (yr) & $46.7 \pm 6.5$ & $48.9 \pm 7.7$ & $51.2 \pm 8.4$ & $53.9 \pm 9.0$ & $56.0 \pm 8.4$ & $58.2 \pm 7.8$ \\
\hline Female sex & $279(70.3)$ & 1600 (53.1) & 747 (45.9) & $521(45.4)$ & $442(49.7)$ & $147(55.5)$ \\
\hline $\mathrm{SBP}(\mathrm{mmHg})$ & $93.6 \pm 4.3$ & $109.8 \pm 5.4$ & $123.3 \pm 3.0$ & $132.8 \pm 2.9$ & $146.2 \pm 5.5$ & $169.1 \pm 10.5$ \\
\hline $\mathrm{DBP}(\mathrm{mmHg})$ & $64.3 \pm 5.7$ & $75.1 \pm 6.7$ & $82.7 \pm 6.7$ & $88.3 \pm 7.3$ & $94.4 \pm 8.4$ & $101.9 \pm 11.1$ \\
\hline Body mass index $\left(\mathrm{kg} / \mathrm{m}^{2}\right)$ & $23.1 \pm 2.5$ & $24.0 \pm 2.9$ & $24.6 \pm 3.1$ & $24.7 \pm 3.1$ & $25.0 \pm 3.3$ & $25.0 \pm 3.4$ \\
\hline \multicolumn{7}{|l|}{ Education } \\
\hline Low & $67(16.9)$ & $672(22.4)$ & $498(30.9)$ & $473(41.5)$ & $423(48.0)$ & $146(57.3)$ \\
\hline Intermediate & $84(21.2)$ & $712(23.7)$ & $389(24.1)$ & $249(21.8)$ & $195(22.1)$ & $56(22.0)$ \\
\hline High & 245 (61.9) & $1,614(53.8)$ & $724(44.9)$ & $418(36.7)$ & $264(29.9)$ & $53(20.8)$ \\
\hline \multicolumn{7}{|l|}{ Income } \\
\hline Low & $57(14.4)$ & $741(25.0)$ & $530(33.2)$ & $467(41.5)$ & $416(48.1)$ & $154(59.7)$ \\
\hline Intermediate & $116(29.3)$ & $915(30.8)$ & $503(31.5)$ & $316(28.1)$ & $242(28.0)$ & $62(24.0)$ \\
\hline High & $223(56.3)$ & $1,310(44.2)$ & $562(35.2)$ & $343(30.5)$ & $206(23.8)$ & $42(16.3)$ \\
\hline Married & $392(98.7)$ & $2,963(98.8)$ & $1,596(98.5)$ & $1,120(98.9)$ & $874(99.1)$ & $257(98.5)$ \\
\hline \multicolumn{7}{|l|}{ Smoking } \\
\hline Never & $281(70.8)$ & $1,776(58.9)$ & $913(56.1)$ & $627(54.6)$ & $520(58.5)$ & $167(63.0)$ \\
\hline Former & $31(7.8)$ & $430(14.3)$ & $265(16.3)$ & $212(18.5)$ & $144(16.2)$ & $31(11.7)$ \\
\hline Current & $85(21.4)$ & $810(26.9)$ & $450(27.6)$ & 309 (26.9) & $225(25.3)$ & $67(25.3)$ \\
\hline Diabetes mellitus & $9(2.3)$ & $122(4.0)$ & $89(5.5)$ & $87(7.6)$ & $55(6.2)$ & $16(6.0)$ \\
\hline Dyslipidemia & $9(2.3)$ & $69(2.3)$ & $29(1.8)$ & $20(1.7)$ & $21(2.4)$ & $2(0.8)$ \\
\hline Fasting glucose (mg/dL) & $81.8 \pm 18.2$ & $85.2 \pm 19.0$ & $86.7 \pm 21.0$ & $87.5 \pm 18.3$ & $89.4 \pm 22.9$ & $90.8 \pm 23.4$ \\
\hline Creatinine $(\mathrm{mg} / \mathrm{dL})$ & $0.8 \pm 0.2$ & $0.8 \pm 0.2$ & $0.8 \pm 0.2$ & $0.8 \pm 0.2$ & $0.8 \pm 0.2$ & $0.8 \pm 0.2$ \\
\hline eGFR $\left(\mathrm{mL} / \mathrm{min} / 1.73 \mathrm{~m}^{2}\right)$ & $96.7 \pm 13.4$ & $94.6 \pm 13.2$ & $93.2 \pm 13.2$ & $92.4 \pm 12.2$ & $91.1 \pm 12.2$ & $89.7 \pm 12.4$ \\
\hline Total cholesterol (mg/dL) & $181.4 \pm 30.5$ & $188.1 \pm 33.9$ & $190.3 \pm 34.6$ & $191.6 \pm 35.9$ & $194.1 \pm 36.8$ & $191.8 \pm 38.1$ \\
\hline Triglyceride $(\mathrm{mg} / \mathrm{dL})$ & $127.4 \pm 73.0$ & $145.0 \pm 91.9$ & $164.8 \pm 108.8$ & $172.4 \pm 106.1$ & $179.2 \pm 119.2$ & $180.0 \pm 107.5$ \\
\hline $\mathrm{HDL}-\mathrm{C}(\mathrm{mg} / \mathrm{dL})$ & $45.9 \pm 9.8$ & $44.9 \pm 9.7$ & $44.4 \pm 9.9$ & $44.7 \pm 10.3$ & $45.3 \pm 10.4$ & $45.8 \pm 11.3$ \\
\hline LDL-C (mg/dL) & $110.1 \pm 28.6$ & $114.4 \pm 31.4$ & $113.4 \pm 32.1$ & $112.7 \pm 33.2$ & $113.7 \pm 33.9$ & $110.5 \pm 35.7$ \\
\hline Albumin (g/dL) & $4.2 \pm 0.3$ & $4.2 \pm 0.3$ & $4.3 \pm 0.3$ & $4.2 \pm 0.3$ & $4.3 \pm 0.3$ & $4.2 \pm 0.3$ \\
\hline Hemoglobin (g/dL) & $12.9 \pm 1.5$ & $13.5 \pm 1.6$ & $13.8 \pm 1.6$ & $13.8 \pm 1.6$ & $13.8 \pm 1.5$ & $13.6 \pm 1.5$ \\
\hline
\end{tabular}

Data are expressed as counts (\%) for categorical variables and as means \pm standard deviations for continuous variables.

DBP, diastolic blood pressure; eGFR, estimated glomerular filtration rate; HDL-C, high-density lipoprotein cholesterol; LDL-C, low-density lipoprotein cholesterol; SBP, systolic blood pressure.

the groups using DBP 70-79 $\mathrm{mmHg}$ as the reference, all the DBP groups $\geq 80 \mathrm{mmHg}$ showed a significantly lower cumulative CKD-free survival rate (log-rank test $\mathrm{p}<0.001$ for all) (Fig. 1B). In the multivariable Cox proportional hazard regression models with baseline data, both $\mathrm{SBP} \geq 120$ $\mathrm{mmHg}$ and SBP < $100 \mathrm{mmHg}$ (HR, 1.66; 95\% CI, 1.08-2.54; $\mathrm{p}=0.02)$ were associated with a significantly increased CKD risk compared with the SBP 100-119 mmHg group (Table 2). Similarly, a significantly increased risk of CKD was observed in the DBP $\geq 80 \mathrm{mmHg}$ and $\mathrm{DBP}<70 \mathrm{mmHg}$ groups (HR, 1.44; 95\% CI, 1.04-2.01; $\mathrm{p}=0.03$ ) compared with the DBP 70-79 mmHg group. However, when we treated $\mathrm{BP}$ as a time-varying variable, the increased CKD risk in the lowest BP group disappeared. In the time-varying multivariable Cox analysis, significant increases in CKD risk were observed only with SBP 130-139 mmHg (HR, 1.39; 95\% CI, 1.10-1.77; $\mathrm{p}=0.007$ ), 140-159 mmHg (HR, 1.79; 95\% CI, 1.40-2.28; $\mathrm{p}<0.001$ ), and $\geq 160 \mathrm{mmHg}$ (HR, 3.22; $95 \% \mathrm{CI}, 2.35-4.40 ; \mathrm{p}<0.001)$. In the DBP groups, increased CKD risks were observed with DBP 90-99 $\mathrm{mmHg}$ (HR, 1.88; 95\% CI, 1.48-2.37; $\mathrm{p}<0.001)$ and $\geq 100 \mathrm{mmHg}(\mathrm{HR}, 4.30$; 95\% CI, 3.20-5.76; $\mathrm{p}<0.001$ ) (Table 2). The stepwise mod- 

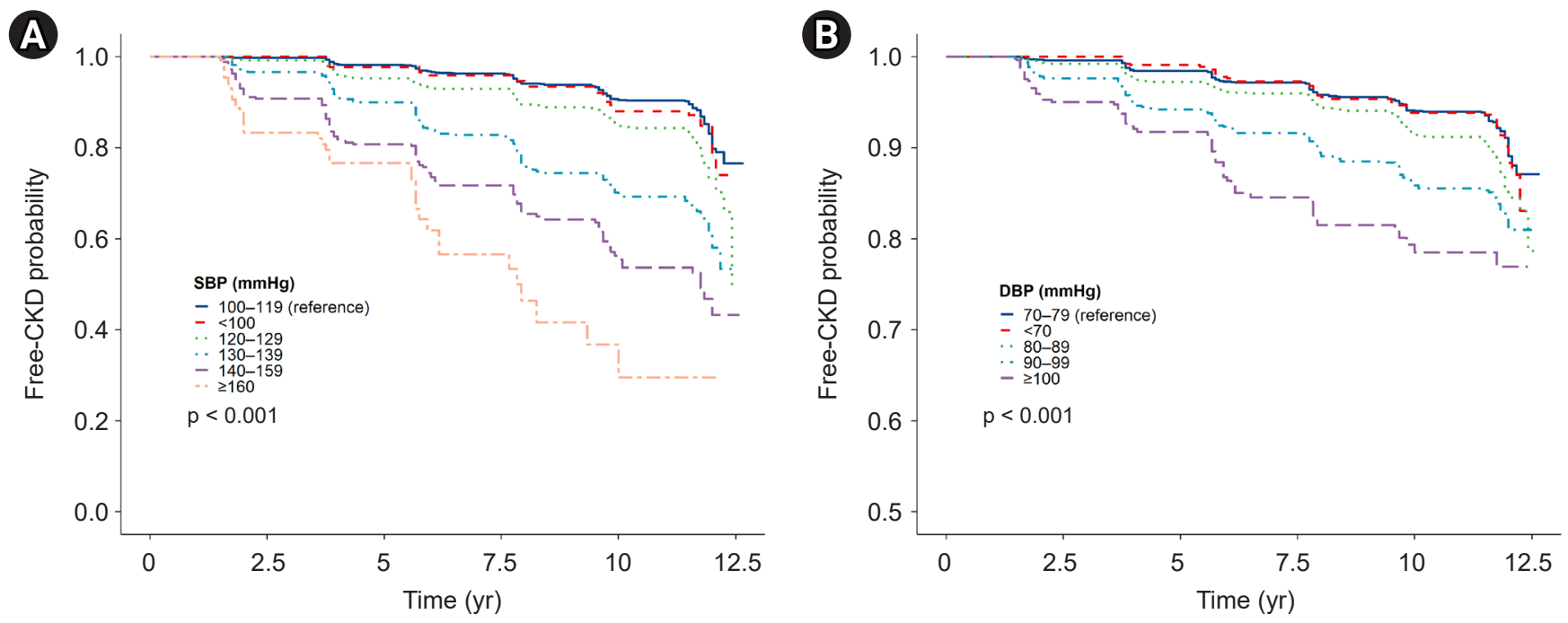

Number at risk

$-\quad 3,016$
$-\quad 397$
$-\quad 1,628$
$-\quad 1,148$
$=\quad 889$
$-\quad 265$

2,789
363
1,468
978
658
148

$\begin{array}{rrr}2,564 & 2,390 & 1,901 \\ 336 & 318 & 226 \\ 1,296 & 1,168 & 826 \\ 791 & 648 & 393 \\ 485 & 356 & 184 \\ 89 & 51 & 19\end{array}$

Number at risk

$\begin{array}{rrrrrrr}- & 2,180 & 1,996 & 1,804 & 1,675 & 1,316 & 9 \\ - & 727 & 671 & 617 & 576 & 470 & 1 \\ - & 2,405 & 2,154 & 1,912 & 1,704 & 1,211 & 10 \\ - & 1,450 & 1,198 & 965 & 801 & 496 & 1 \\ - & 581 & 385 & 263 & 175 & 96 & 0\end{array}$

Figure 1. Kaplan-Meier curves for the development of CKD according to baseline (A) SBP and (B) DBP. The cumulative CKD-free survival rate gradually decreased as BP increased in groups with SBP $\geq 120 \mathrm{mmHg}$ and DBP $\geq 80 \mathrm{mmHg}$ compared with the SBP 100$119 \mathrm{mmHg}$ and DBP 70-79 mmHg groups, respectively.

BP, blood pressure; CKD, chronic kidney disease; DBP, diastolic blood pressure; SBP, systolic blood pressure.

Table 2. Relationship between blood pressure and incident chronic kidney disease

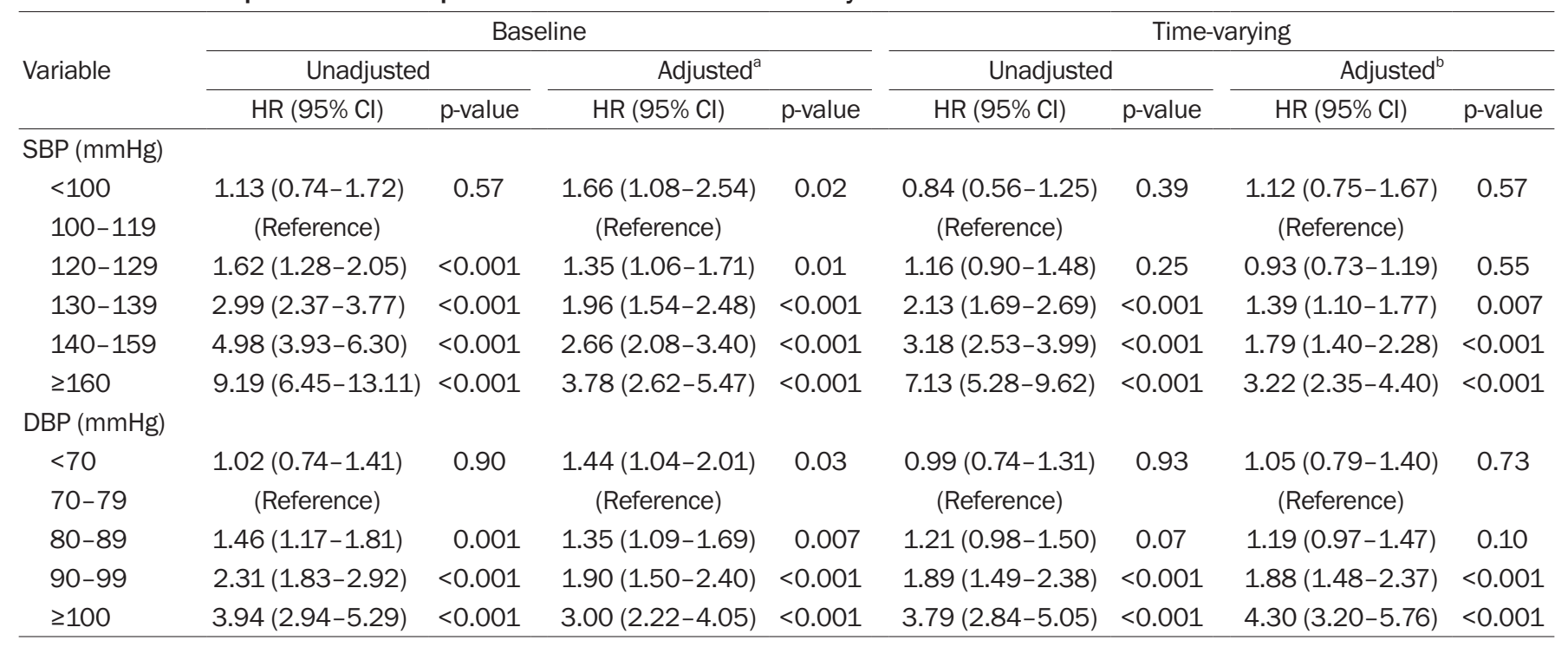

$\mathrm{Cl}$, confidence interval; DBP, diastolic blood pressure; HR, hazard ratio; SBP, systolic blood pressure.

a Baseline data were adjusted for age, sex, educational attainment, income level, smoking status, body mass index (BMI), history of diabetes mellitus (DM) and dyslipidemia, estimated glomerular filtration rate (eGFR), total cholesterol, albumin, and hemoglobin, ${ }^{b}$ Data were adjusted for baseline sex, educational attainment, income level, smoking status, history of DM and dyslipidemia, eGFR, and albumin and time-updated age, BMI, total cholesterol, and hemoglobin. 
eling of these Cox regression analyses is presented in Supplementary Tables 4 and 5 (available online). Collectively, a significantly increased CKD risk was observed in patients with $\mathrm{SBP} \geq 130 \mathrm{mmHg}$ and $\mathrm{DBP} \geq 90 \mathrm{mmHg}$.

\section{Sensitivity analyses}

We further conducted a sensitivity analysis in which baseline and time-varying BPs were treated as continuous variables to assess the consistency of our findings. When evaluating the nonlinear relationship between BP and CKD risk using restricted cubic spline models, the CKD risk increased incrementally with higher baseline and time-varying SBPs (Fig. 2A, B). The CKD risk tended to increase with $\mathrm{SBP}<100 \mathrm{mmHg}$, but that was not significant due to wide CIs. Similarly, CKD hazards gradually increased with higher baseline and time-varying DBPs (Fig. 2C, D). The lowest CKD hazard was shown in people with a time-varying SBP between 100 and $119 \mathrm{mmHg}$, and that CKD hazard was nearly constant in those with a time-varying $\mathrm{DBP}<80$ $\mathrm{mmHg}$.

\section{Subgroup analyses}

In subgroup analyses, we assessed interactions between time-varying BPs and stratified subgroups (time-updated age, sex, time-updated BMI, baseline DM, and time-updated total cholesterol) using a likelihood ratio test. Subjects were categorized into two groups based on SBP $\geq 130$ $\mathrm{mmHg}$ and DBP $\geq 90 \mathrm{mmHg}$, both of which are associated with increased CKD risk. The association of SBP and DBP with incident CKD was consistent across all subgroups (Fig. 3). However, age had a significant interaction with the relationship between time-varying $\mathrm{BP}$ and incident $\mathrm{CKD}$. The CKD risk increased significantly in both age groups ( $<60$ years and $\geq 60$ years), but the risk in the subgroup of patients aged $<60$ years was higher.

\section{Relationship between blood pressure and incident cardio- vascular disease}

Because the BP thresholds associated with an increased CVD risk might differ from those associated with CKD risk, we also analyzed the incident CVD risk according to BP. During follow-up, 404 CVD events (5.5\%) occurred, and the crude incidence rate was 7 (95\% CI, 6-7) per 1,000 person-years. The multivariable Cox proportional hazard regression analysis for incident CVD is presented in Table 3. Consistent with incident CKD, time-varying SBP 130-139 mmHg (HR, 1.61; 95\% CI, 1.19-2.18; p = 0.002), 140-159 mmHg (HR, 2.34; 95\% CI, 1.72-3.17; p <0.001), and $\geq 160$ mmHg (HR, 2.88; 95\% CI, 1.84-4.50; p < 0.001) were significantly associated with increased CVD risk compared with SBP 100-119 mmHg. Results were similar in the DBP groups; a significant increase in incident CVD risk was observed with time-varying DBP 90-99 mmHg (HR, 1.69; 95\% CI, 1.26-2.25; $\mathrm{p}<0.001$ ) and $\geq 100 \mathrm{mmHg}$ (HR, 3.31; 95\% CI, 2.34-4.70; $<<0.001$ ) compared with DBP 70-79 mmHg.

\section{Discussion}

Using data from 7,343 subjects not taking antihypertensive medications, we demonstrated a graded association between time-varying BP and CKD development. Compared with the reference categories of SBP 100-119 $\mathrm{mmHg}$ and DBP 70-79 mmHg, CKD risk increased from SBP $\geq 130$ $\mathrm{mmHg}$ and $\mathrm{DBP} \geq 90 \mathrm{mmHg}$. Those BP thresholds were consistent when evaluating the association between BP and CVD risk. These results indicate that BP control interventions in treatment-naïve individuals should start when the SBP or DBP reaches $130 \mathrm{mmHg}$ or $90 \mathrm{mmHg}$, respectively, to prevent CVD and CKD.

Several observational studies have shown that an increase in SBP is associated with a substantial risk of CKD in the general population [11,18-23]. However, considerable uncertainty remains about the SBP level that leads to CKD. The SBP threshold associated with increased CKD risk has been reported as $120-140 \mathrm{mmHg}$ in previous studies. This inconsistency among studies can largely be explained by differences in the baseline characteristics of the various study populations. Importantly, because the aforementioned studies included subjects without regard to the use of antihypertensive medications, a substantial proportion of those subjects used antihypertensive drugs at baseline or during follow-up. It is noteworthy that the relationship between BP and patient outcomes can vary depending on the use of antihypertensive medications. A previous study reported that the association between $\mathrm{BP}$ and cardiorenal death was J-shaped in users of antihypertensive medications, whereas the association was linear in non-users [24]. 

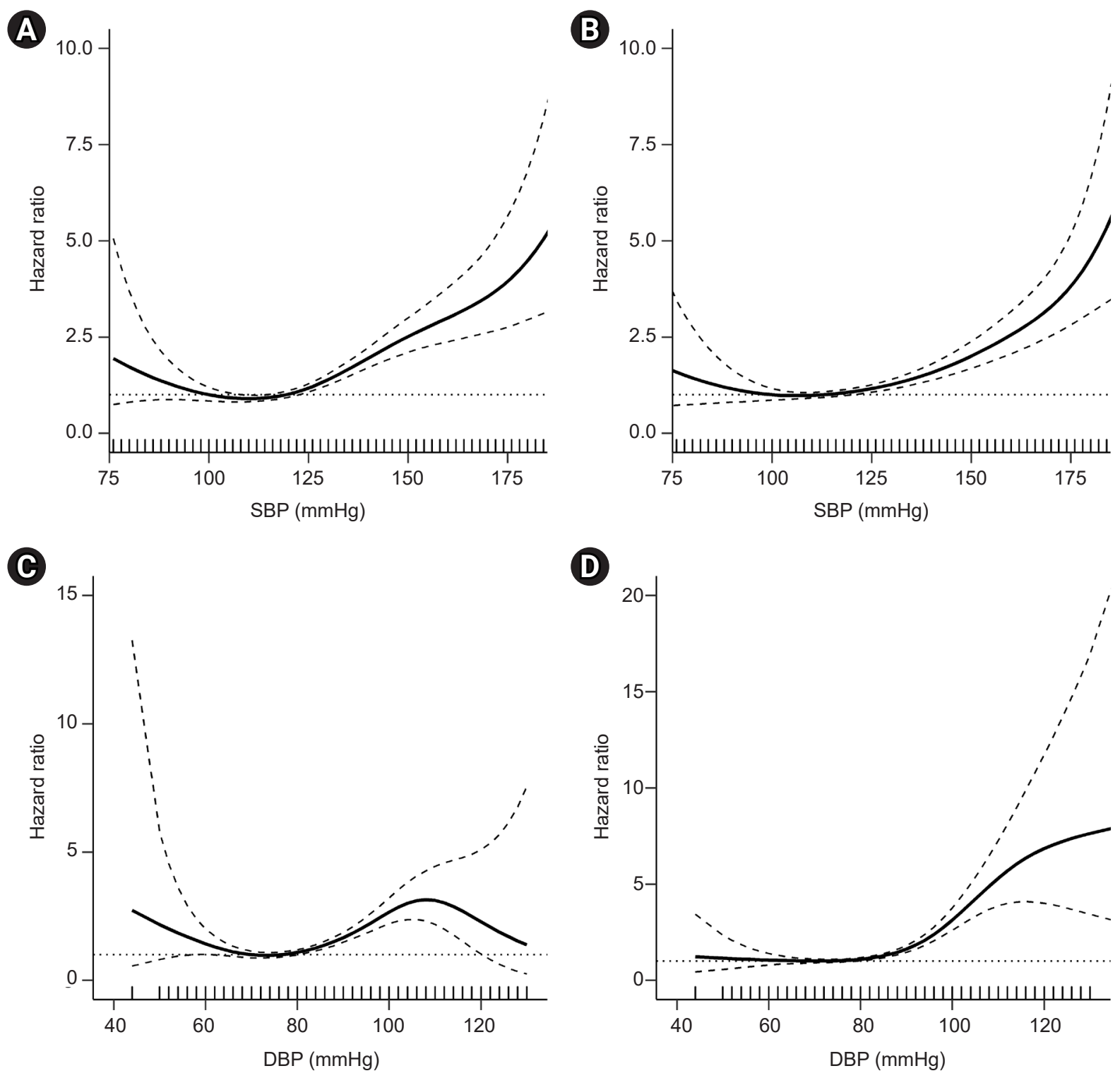

Figure 2. Restricted cubic spline curve for the incident CKD risk according to (A) baseline SBP, (B) time-updated SBP, (C) baseline DBP, and (D) time-updated DBP. All of the curves show an increased risk of CKD with higher BPs, and those relationships are clearer with time-varying BPs. Baseline data were adjusted for age, sex, educational attainment, income level, smoking status, BMI, history of DM and dyslipidemia, eGFR, total cholesterol, albumin, and hemoglobin levels. Time-updated data were adjusted for baseline sex, educational attainment, income level, smoking status, history of DM and dyslipidemia, eGFR, and albumin and time-updated age, BMI, total cholesterol, and hemoglobin.

Solid line, estimated hazard ratio; dashed line, 95\% confidence interval; dotted line, hazard ratio of 1. BMI, body mass index; BP, blood pressure; CKD, chronic kidney disease; DBP, diastolic blood pressure; DM, diabetes mellitus; eGFR, estimated glomerular filtration rate; SBP, systolic blood pressure.

In that study, the risk of end-stage kidney disease was significantly higher in antihypertensive users than in non-users, even if they were in the same BP categories. Therefore, previous observational studies cannot be used to identify a BP threshold associated with an increased CKD risk in treatment-naïve individuals. In this study, we began by excluding patients taking antihypertensive medications at baseline. However, excluding all subjects who started tak- 
(A)

Subgroup

HR $(95 \% \mathrm{Cl}) \quad \mathrm{p}$ for

interaction

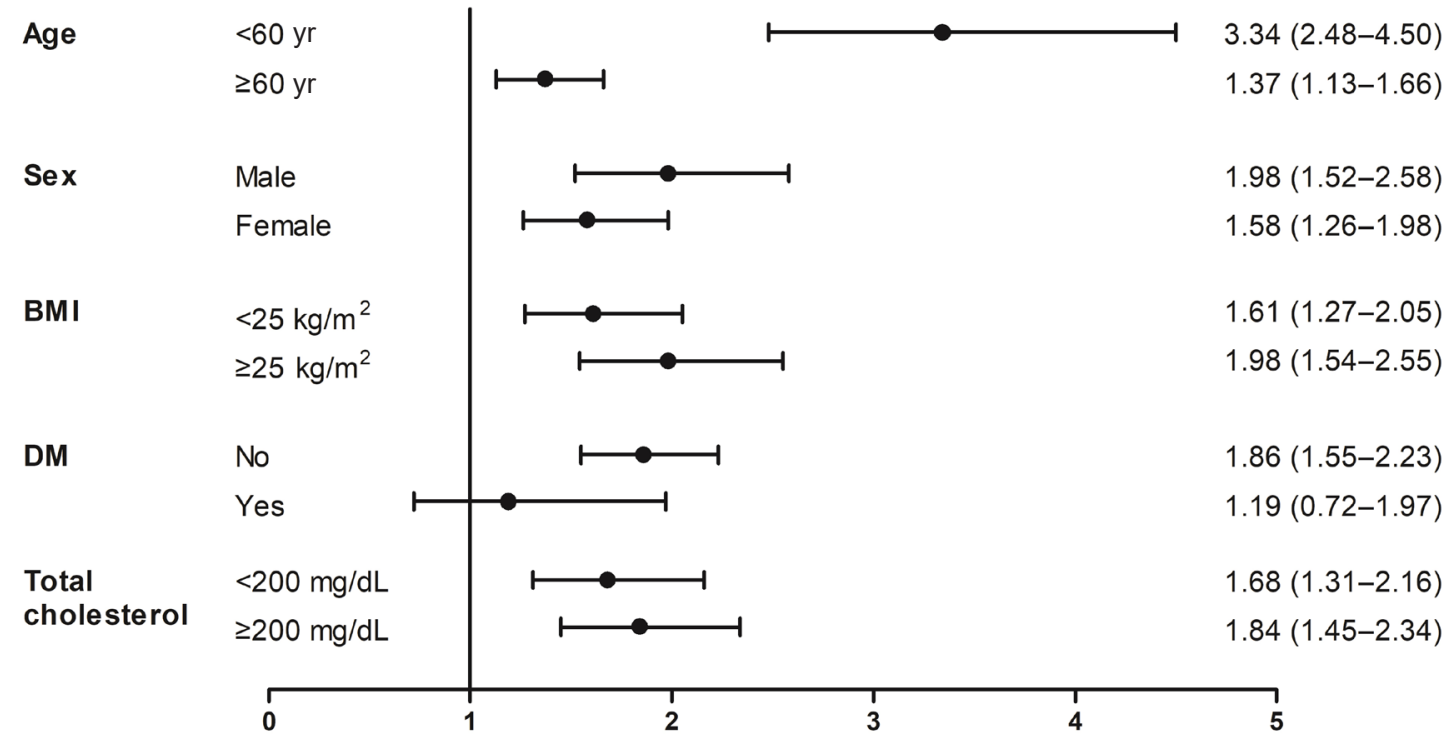

B

Subgroup

$\mathrm{HR}(95 \% \mathrm{Cl}) \quad \mathrm{p}$ for

interaction

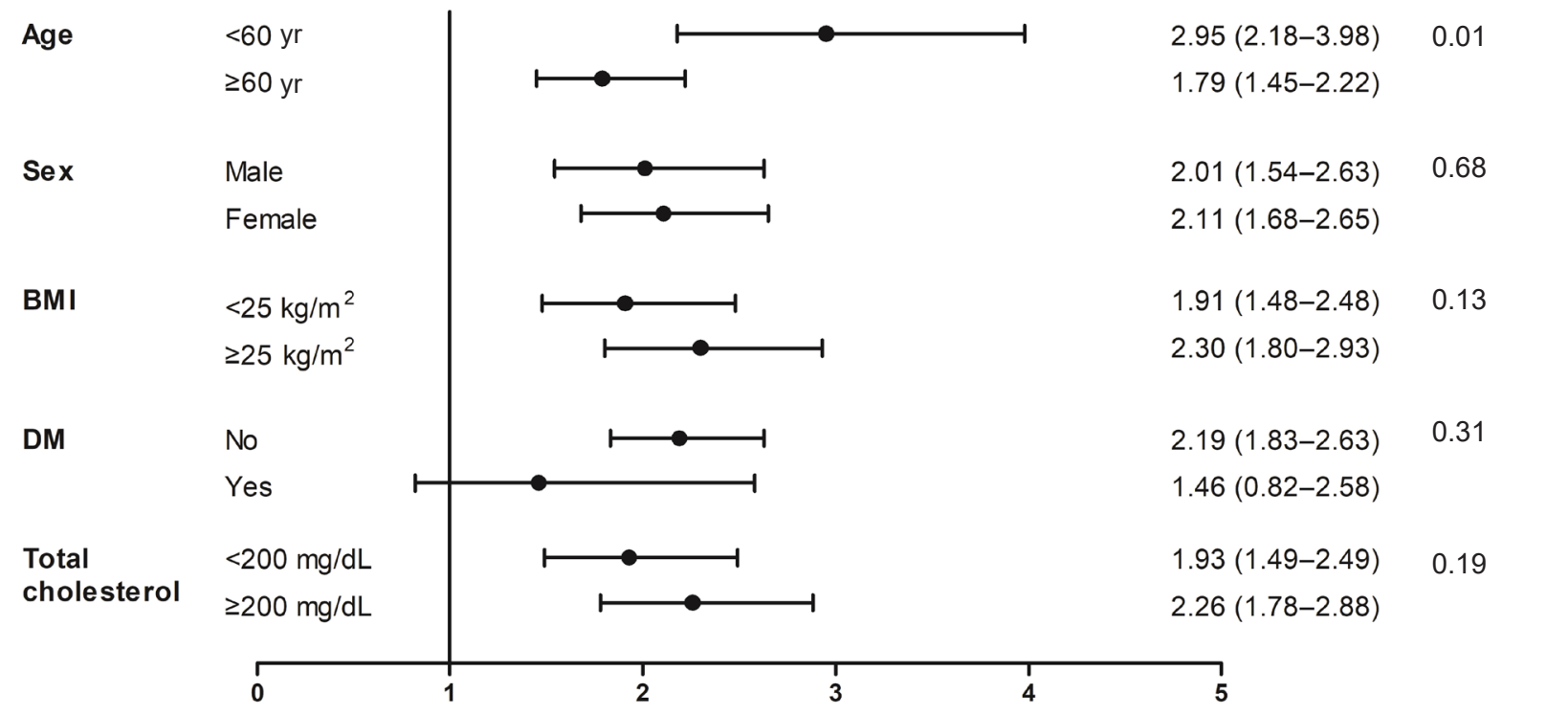

Figure 3. Subgroup analyses and interaction terms for incident CKD according to time-updated (A) SBP and (B) DBP. The association between incident CKD and time-varying BP showed a similar pattern independent of sex, time-updated BMI, history of DM, and time-updated total cholesterol, but not independent of age. The risk of incident CKD was greater among those aged $<60$ years than among those aged $\geq 60$ years. Data were adjusted for baseline sex, educational attainment, income level, smoking status, history of DM and dyslipidemia, eGFR, and albumin and time-updated age, BMI, total cholesterol, and hemoglobin. Forest plots show the HR (dots) and $95 \% \mathrm{Cl}$ (horizontal bars) of time-updated BP (SBP $\geq 130 \mathrm{mmHg}$ and DBP $\geq 90 \mathrm{mmHg}$ ) for incident CKD in the subgroups defined by demographic and clinical characteristics.

$\mathrm{BMI}$, body mass index; $\mathrm{BP}$, blood pressure; $\mathrm{Cl}$, confidence interval; CKD, chronic kidney disease; DBP, diastolic blood pressure; DM, diabetes mellitus; eGFR, estimated glomerular filtration rate; HR, hazard ratio; SBP, systolic blood pressure. 
Table 3. Relationship between blood pressure and incident cardiovascular disease

\begin{tabular}{|c|c|c|c|c|c|c|c|c|}
\hline \multirow{3}{*}{ Variable } & \multicolumn{4}{|c|}{ Baseline } & \multicolumn{4}{|c|}{ Time-varying } \\
\hline & \multicolumn{2}{|c|}{ Unadjusted } & \multicolumn{2}{|l|}{ Adjusted $^{a}$} & \multicolumn{2}{|l|}{ Unadjusted } & \multicolumn{2}{|l|}{ Adjusted $^{b}$} \\
\hline & $\mathrm{HR}(95 \% \mathrm{Cl})$ & p-value & $\mathrm{HR}(95 \% \mathrm{Cl})$ & p-value & $\operatorname{HR}(95 \% \mathrm{Cl})$ & $\mathrm{p}$-value & $\mathrm{HR}(95 \% \mathrm{Cl})$ & p-value \\
\hline \multicolumn{9}{|l|}{$\mathrm{SBP}(\mathrm{mmHg})$} \\
\hline$<100$ & $0.31(0.13-0.76)$ & 0.01 & $0.40(0.16-0.99)$ & 0.05 & $0.56(0.31-1.02)$ & 0.06 & $0.69(0.38-1.26)$ & 0.23 \\
\hline $100-119$ & (Reference) & & (Reference) & & (Reference) & & (Reference) & \\
\hline $120-129$ & $1.63(1.24-2.14)$ & $<0.001$ & $1.36(1.03-1.79)$ & 0.03 & $1.51(1.14-2.00)$ & 0.004 & $1.26(0.95-1.69)$ & 0.11 \\
\hline $130-139$ & $2.81(2.14-3.69)$ & $<0.001$ & $1.93(1.45-2.55)$ & $<0.001$ & $2.20(1.65-2.94)$ & $<0.001$ & $1.61(1.19-2.18)$ & 0.002 \\
\hline $140-159$ & $3.51(2.61-4.72)$ & $<0.001$ & $2.11(1.55-2.89)$ & $<0.001$ & $3.39(2.57-4.48)$ & $<0.001$ & $2.34(1.72-3.17)$ & $<0.001$ \\
\hline$\geq 160$ & 6.19 (3.97-9.65) & $<0.001$ & $3.19(2.01-5.05)$ & $<0.001$ & $4.58(2.98-7.06)$ & $<0.001$ & $2.88(1.84-4.50)$ & $<0.001$ \\
\hline \multicolumn{9}{|l|}{$\mathrm{DBP}(\mathrm{mmHg})$} \\
\hline$<70$ & $0.64(0.40-1.02)$ & 0.06 & $0.79(0.50-1.27)$ & 0.33 & $0.71(0.48-1.05)$ & 0.08 & $0.80(0.54-1.19)$ & 0.27 \\
\hline $70-79$ & (Reference) & & (Reference) & & (Reference) & & (Reference) & \\
\hline $80-89$ & $1.28(0.98-1.67)$ & 0.07 & $1.16(0.89-1.51)$ & 0.29 & $1.15(0.89-1.49)$ & 0.28 & $1.04(0.80-1.35)$ & 0.76 \\
\hline $90-99$ & $2.18(1.66-2.87)$ & $<0.001$ & $1.70(1.29-2.26)$ & $<0.001$ & $1.93(1.46-2.55)$ & $<0.001$ & $1.69(1.26-2.25)$ & $<0.001$ \\
\hline$\geq 100$ & $3.49(2.47-4.94)$ & $<0.001$ & $2.49(1.74-3.55)$ & $<0.001$ & $3.92(2.81-5.47)$ & $<0.001$ & $3.31(2.34-4.70)$ & $<0.001$ \\
\hline
\end{tabular}

$\mathrm{Cl}$, confidence interval; DBP, diastolic blood pressure; HR, hazard ratio; SBP, systolic blood pressure.

a Baseline data were adjusted for age, sex, educational attainment, income level, smoking status, body mass index (BMI), history of diabetes mellitus (DM) and dyslipidemia, estimated glomerular filtration rate (eGFR), total cholesterol, albumin, and hemoglobin. ${ }^{b}$ Data were adjusted for baseline sex, educational attainment, income level, smoking status, history of DM and dyslipidemia, eGFR, and albumin and time-updated age, BMI, total cholesterol, and hemoglobin.

ing antihypertensive medication during follow-up can lead to selection bias. Therefore, we included those subjects at baseline and censored them when they started taking antihypertensive medications during follow-up. As a result, we found that $\mathrm{SBP} \geq 130 \mathrm{mmHg}$ and $\mathrm{DBP} \geq 90 \mathrm{mmHg}$ are indications for HTN management to prevent CKD in treatment-naïve individuals.

The relationship between baseline BP and CKD was J-shaped; however, after accounting for time-varying risk factors, that association disappeared, and CKD risk remained similar among subjects with BP lower than the threshold values. A J- or U-shaped relationship between BP values and CVD was previously reported $[25,26]$. In addition, some studies have shown a linear association between BP and CKD $[23,24]$, whereas other studies found that those with low BP did not have a lower risk of CKD [21,27]. The high risk of CKD or CVD in people with low BP could be a result of a cardiovascular burden that produces both low BP and adverse outcomes. In this study, we included subjects with a low CVD risk and excluded those with a history of CVD or CKD. Importantly, a meta-analysis of 7 RCTs using time-updated BP values showed that the J- shaped association between low BP at baseline and mortality during follow-up was explained by poor health conditions, not BP-specific events [28]. In our study, the low SBP $(<100$ $\mathrm{mmHg}$ ) group had lower BMI, albumin, and hemoglobin levels than the subjects in other groups, indicating poor nutritional status. Thus, although the detrimental nutritional status could contribute to low baseline BP and a high risk of CKD, a time-dependent analysis might mitigate the confounding effect of nutritional status on the relationship between BP and CKD. This discrepancy between baseline and time-varying BPs in our study was in agreement with the results of a previous large observational study conducted in Hong Kong [29]. Nevertheless, our results do not support the linear association between BP and CKD presented in large observational studies $[23,24]$. Therefore, further large observational studies and meta-analyses are needed to identify whether 'the lower the better' concept can be applied to treatment-naïve individuals with a low risk of CVD.

Although high SBP has been reported to be associated with both renal and cardiovascular complications [30-32], reports regarding its effects on CKD and CVD risks have conflicted. The SPRINT study reported that intensive SBP control, targeted to $120 \mathrm{mmHg}$, was associated with a reduction in the risk of CVD [8]. However, no favorable effect of intensive BP control on CVD events was found in the 
Action to Control Cardiovascular Risk in Diabetes (ACCORD) study [9]. Importantly, both SPRINT and ACCORD reported that more patients without baseline CKD in the intensive SBP control group experienced eGFR reduction [10]. However, those results cannot be applied to treatment-naïve individuals because those RCTs were conducted for patients with a high CVD risk who had already taken antihypertensive medications. We examined the relationship between BP and CVD as a secondary outcome in our study and found that the BP thresholds associated with increased CKD and CVD risks were the same: SBP $\geq 130$ $\mathrm{mmHg}$ and $\mathrm{DBP} \geq 90 \mathrm{mmHg}$. These findings suggest that $\mathrm{SBP} \geq 130 \mathrm{mmHg}$ or DBP $\geq 90 \mathrm{mmHg}$ should be the points to begin BP control intervention in treatment-naïve individuals. Due to the nature of our study, we cannot provide an 'optimal target' BP that will reduce CKD or CVD risks. Therefore, further RCTs are warranted to establish appropriate $\mathrm{BP}$ targets for reducing both CKD and CVD risk in the treatment-naïve population.

In our study, the relationship between SBP and CKD was more pronounced in participants younger than 60 years than in those older than 60 years. Although the prevalence of HTN and CKD increases with age [33,34], conflicting results have been reported about the age-specific relationship between CKD and SBP. Previous studies have reported that older patients with HTN had a prominent [35], similar [11], or attenuated $[36,37]$ risk of CKD compared with younger patients. This discrepancy might be caused by differences in patient characteristics, statistical methods, or whether the use of antihypertensive medication was considered. Interestingly, previous large cohort studies have reported that the relationships between $\mathrm{BP}$ and several CVD outcomes were robust in young adults and tended to attenuate with increasing age $[1,36,38]$. Therefore, because CKD and CVD share common risk factors, the age-specific risk of CKD might be similar to the risk of CVD. The high risk of CKD in subjects $<60$ years might be caused by low awareness and treatment rates of HTN [39]. However, we excluded subjects treated with antihypertensive medication at baseline and censored those newly treated during the follow-up period. Therefore, our findings indicate that more efforts should be made to improve the detection and management of HTN, especially in people aged $<60$ years.

The strengths of our study include a well-validated, representative cohort consisting only of individuals not taking antihypertensive medication. This stringent approach helped us to show a correct and natural course of CKD development in subjects who had not taken antihypertensive medications. Moreover, our cohort had a long follow-up duration, and changes in risk factors over time were considered through the use of time-varying covariates. Nevertheless, this study also has some limitations. First, our observational study cannot exclude the possibility of residual confounding by unmeasured variables, despite our comprehensive adjustment for confounders. Second, some misclassification could arise because BP was measured biennially and was not measured using 24hour ambulatory monitoring [40], and the assessment of antihypertensive and CVD medication intake was based on participants' self-report questionnaires. However, the use of time-varying covariates at least partly compensates for the possibility of those measurement errors. Third, we had no data to explain why participants did not take antihypertensive medications, even though they met the criteria for HTN. They might have been advised to make lifestyle modifications, have declined medication intake, or been lost to follow up. Finally, our results might not be applicable to other countries with various races and socioeconomic statuses because our study involved only Koreans.

In conclusion, an SBP $\geq 130 \mathrm{mmHg}$ or $\mathrm{DBP} \geq 90 \mathrm{mmHg}$ was associated with an increased risk of incident CKD in treatment-naïve individuals. Therefore, BP-lowering interventions should be considered when BP exceeds those thresholds to prevent CKD in treatment-naïve individuals.

\section{Conflicts of interest}

All authors have no conflicts of interest to declare.

\section{Funding}

This research was supported by the Soonchunhyang University Research Fund (20200027).

\section{Acknowledgments}

The authors thank the National Research Institute of Health for access to KoGES data. 


\section{Authors' contributions}

\author{
Conceptualization: $\mathrm{HK}, \mathrm{DCH}$ \\ Data curation: HK, HL, SHK \\ Formal analysis, Visualization: HK, HL, JSJ \\ Funding acquisition: HK \\ Methodology: HK, HL, HN \\ Project administration: $\mathrm{HK}$ \\ Writing-Original Draft: HL
}

\section{ORCID}

Haekyung Lee, https://orcid.org/0000-0001-5211-3961

Soon Hyo Kwon, https://orcid.org/0000-0002-4114-4196

Jin Seok Jeon, https://orcid.org/0000-0003-2421-2289

Hyunjin Noh, https://orcid.org/0000-0002-1904-1684

Dong Cheol Han, https://orcid.org/0000-0002-8835-8642

Hyoungnae Kim, https://orcid.org/0000-0002-5359-0214

\section{References}

1. Rapsomaniki E, Timmis A, George J, et al. Blood pressure and incidence of twelve cardiovascular diseases: lifetime risks, healthy life-years lost, and age-specific associations in 1.25 million people. Lancet 2014;383:1899-1911.

2. GBD 2017 Risk Factor Collaborators. Global, regional, and national comparative risk assessment of 84 behavioural, environmental and occupational, and metabolic risks or clusters of risks for 195 countries and territories, 1990-2017: a systematic analysis for the Global Burden of Disease Study 2017. Lancet 2018;392:1923-1994.

3. Jha V, Garcia-Garcia G, Iseki K, et al. Chronic kidney disease: global dimension and perspectives. Lancet 2013;382:260-272.

4. Jin DC, Yun SR, Lee SW, et al. Current characteristics of dialysis therapy in Korea: 2016 registry data focusing on diabetic patients. Kidney Res Clin Pract 2018;37:20-29.

5. Lash JP, Go AS, Appel LJ, et al. Chronic Renal Insufficiency Cohort (CRIC) Study: baseline characteristics and associations with kidney function. Clin J Am Soc Nephrol 2009;4:1302-1311.

6. Rao MV, Qiu Y, Wang C, Bakris G. Hypertension and CKD: Kidney Early Evaluation Program (KEEP) and National Health and Nutrition Examination Survey (NHANES), 1999-2004. Am J Kidney Dis 2008;51(4 Suppl 2):S30-S37.

7. Whelton PK, Carey RM, Aronow WS, et al. 2017 ACC/AHA/ AAPA/ABC/ACPM/AGS/APhA/ASH/ASPC/NMA/PCNA guide- line for the prevention, detection, evaluation, and management of high blood pressure in adults: a report of the American College of Cardiology/American Heart Association Task Force on Clinical Practice Guidelines. Hypertension 2018;71:e13-e115.

8. SPRINT Research Group; Wright JT Jr, Williamson JD, et al. A randomized trial of intensive versus standard blood-pressure control. N Engl J Med 2015;373:2103-2116.

9. ACCORD Study Group; Cushman WC, Evans GW, et al. Effects of intensive blood-pressure control in type 2 diabetes mellitus. $N$ Engl J Med 2010;362:1575-1585.

10. Beddhu S, Greene T, Boucher R, et al. Intensive systolic blood pressure control and incident chronic kidney disease in people with and without diabetes mellitus: secondary analyses of two randomized controlled trials. Lancet Diabetes Endocrinol 2018;6:555-563.

11. Kanno A, Kikuya M, Ohkubo T, et al. Pre-hypertension as a significant predictor of chronic kidney disease in a general population: the Ohasama Study. Nephrol Dial Transplant 2012;27:3218-3223.

12. Xue H, Wang J, Hou J, et al. Prehypertension and chronic kidney disease in chinese population: four-year follow-up study. PLoS One 2015;10:e144438.

13. Higashikuni Y, Ishizaka N, Ishizaka Y, Toda E, Nagai R, Yamakado M. Relationship between blood pressure and chronic kidney disease in the Japanese population: the lower the better even in individuals without hypertension? Hypertens Res 2008;31:213219.

14. Wen J, Hao J, Zhang Y, et al. Risk scores for predicting incident chronic kidney disease among rural Chinese people: a village-based cohort study. BMC Nephrol 2020;21:120.

15. Lim S, Jang HC, Lee HK, Kimm KC, Park C, Cho NH. A rural-urban comparison of the characteristics of the metabolic syndrome by gender in Korea: the Korean Health and Genome Study (KHGS). J Endocrinol Invest 2006;29:313-319.

16. Kim Y, Han BG; KoGES group. Cohort profile: the Korean Genome and Epidemiology Study (KoGES) Consortium. Int J Epidemiol 2017;46:e20.

17. Levey AS, Stevens LA, Schmid CH, et al. A new equation to estimate glomerular filtration rate. Ann Intern Med 2009;150:604612.

18. Schaeffner ES, Kurth T, Bowman TS, Gelber RP, Gaziano JM. Blood pressure measures and risk of chronic kidney disease in men. Nephrol Dial Transplant 2008;23:1246-1251.

19. Yano Y, Fujimoto S, Sato Y, et al. Association between prehypertension and chronic kidney disease in the Japanese general 
population. Kidney Int 2012;81:293-299.

20. Maeda T, Yoshimura C, Takahashi K, et al. Usefulness of the blood pressure classification in the new 2017 ACC/AHA hypertension guidelines for the prediction of new-onset chronic kidney disease. J Hum Hypertens 2019;33:873-878.

21. Lohr JW, Golzy M, Carter RL, Arora P. Elevated systolic blood pressure is associated with increased incidence of chronic kidney disease but not mortality in elderly veterans. J Am Soc Hypertens 2015;9:29-37.

22. Tozawa M, Iseki K, Iseki C, Kinjo K, Ikemiya Y, Takishita S. Blood pressure predicts risk of developing end-stage renal disease in men and women. Hypertension 2003;41:1341-1345.

23. Chang TI, Lim H, Park CH, et al. Associations of systolic blood pressure with incident CKD G3-G5: a cohort study of South Korean adults. Am J Kidney Dis 2020;76:224-232.

24. Jung HH, Park JI, Jeong JS. Blood pressure-related risk among users versus nonusers of antihypertensives: a population-based cohort in Korea. Hypertension 2018;71:1047-1055.

25. Sundström J, Sheikhi R, Ostgren CJ, et al. Blood pressure levels and risk of cardiovascular events and mortality in type-2 diabetes: cohort study of 34009 primary care patients. J Hypertens 2013;31:1603-1610.

26. Kontopantelis E, Springate DA, Reeves D, et al. Glucose, blood pressure and cholesterol levels and their relationships to clinical outcomes in type 2 diabetes: a retrospective cohort study. Diabetologia 2015;58:505-518.

27. Hanratty R, Chonchol M, Havranek EP, et al. Relationship between blood pressure and incident chronic kidney disease in hypertensive patients. Clin J Am Soc Nephrol 2011;6:2605-2611.

28. Boutitie F, Gueyffier F, Pocock S, Fagard R, Boissel JP; INDANA Project Steering Committee. INdividual Data ANalysis of Antihypertensive intervention: J-shaped relationship between blood pressure and mortality in hypertensive patients: new insights from a meta-analysis of individual-patient data. Ann Intern Med 2002;136:438-448.

29. Wan EY, Yu EY, Chin WY, Fong DY, Choi EP, Lam CL. Association of blood pressure and risk of cardiovascular and chronic kidney disease in Hong Kong hypertensive patients. Hypertension
2019;74:331-340.

30. Hsu CY, McCulloch CE, Darbinian J, Go AS, Iribarren C. Elevated blood pressure and risk of end-stage renal disease in subjects without baseline kidney disease. Arch Intern Med 2005;165:923928.

31. Stokes J 3rd, Kannel WB, Wolf PA, D'Agostino RB, Cupples LA. Blood pressure as a risk factor for cardiovascular disease: the Framingham study: 30 years of follow-up. Hypertension 1989;13(5 Suppl):I13-I18.

32. Go AS, Chertow GM, Fan D, McCulloch CE, Hsu CY. Chronic kidney disease and the risks of death, cardiovascular events, and hospitalization. N Engl J Med 2004;351:1296-1305.

33. Virani SS, Alonso A, Benjamin EJ, et al. Heart disease and stroke statistics-2020 update: a report from the American Heart Association. Circulation 2020;141:e139-e596.

34. Coresh J, Selvin E, Stevens LA, et al. Prevalence of chronic kidney disease in the United States. JAMA 2007;298:2038-2047.

35. Garofalo C, Borrelli S, Pacilio M, et al. Hypertension and prehypertension and prediction of development of decreased estimated GFR in the general population: a meta-analysis of cohort studies. Am J Kidney Dis 2016;67:89-97.

36. Lacey B, Lewington S, Clarke R, et al. Age-specific association between blood pressure and vascular and non-vascular chronic diseases in 0.5 million adults in China: a prospective cohort study. Lancet Glob Health 2018;6:e641-e649.

37. Kim YT, Chung HJ, Park BR, et al. Risk of cardiovascular disease and chronic kidney disease according to 2017 blood pressure categories in diabetes mellitus. Hypertension 2020;76:766-775.

38. Kim H, Lee S, Ha E, et al. Age and sex specific target of blood pressure for the prevention of cardiovascular event among the treatment naive hypertensive patients. Sci Rep 2020;10:21538.

39. Kang SH, Kim SH, Cho JH, et al. Prevalence, awareness, treatment, and control of hypertension in Korea. Sci Rep 2019;9: 10970.

40. Hansen TW, Kikuya M, Thijs L, et al. Prognostic superiority of daytime ambulatory over conventional blood pressure in four populations: a meta-analysis of 7,030 individuals. J Hypertens 2007;25:1554-1564. 